\title{
Electrochemistry-mass spectrometry: an emerging hyphenated technique for bioanalysis
}

\author{
Martin Vogel • Uwe Karst
}

Published online: 28 February 2012

(C) Springer-Verlag 2012

While the combination of electrochemistry (EC)-mass spectrometry (MS) was introduced several decades ago, its potential to provide valuable information to solve (bio) analytical questions has been exploited only partially to date, and only very recently. This is due to the fact that these two groups of analytical techniques are traditionally used by different scientific communities who rarely overlap. Furthermore, many of its (bio)analytical applications - which are being found at an increasing rate - are not obvious, even to experts in this field. However, there is still plenty of room for technical innovation and improvements in this field.

Challenges on the technical side include integrating electrochemical oxidation directly into the ESI interface in order to monitor the formation of very short-lived intermediates. Closer integration of the electrochemical part into existing liquid chromatography(LC)/MS systems with respect to hardware and software would also significantly increase the use of $\mathrm{EC} / \mathrm{MS}$ in various industries. The vast majority of publications on EC/MS currently use the electrospray ionization (ESI) interface, but the utilisation of other ion sources could greatly expand the range of applications for EC/MS.

The majority of applications of EC/MS are still associated with the fields of pharmaceutical chemistry and drug metab- olism, as EC/MS provides an easy and rapid means to predict possible oxidation products of xenobiotics in the liver. This is particularly true when generating and identifying reactive metabolites, as these tasks are hampered by protein adduct formation in established methods of simulating the oxidation of drugs, such as liver cell microsomes and animal tests. While EC/MS is certainly not able to predict all of the products that arise from oxidative reactions in living organisms, it is a valuable complementary tool in many cases.

More recent applications include the bioaffinity screening of electrogenerated oxidation products, and attempts to predict the allergenic potential of xenobiotics by investigating the peptide and protein adduct formation of their electrogenerated products.

In this special issue, a collection of manuscripts that address several hot topics in EC/MS and have been written by leading authors in this field have been assembled into a dedicated issue for the first time. There are also other signs of expansion in this field, including a recent first international workshop on EC/MS. We look forward to exciting new technical developments in and applications of EC/MS in the near future!

Published in the special paper collection Electrochemistry-Mass Spectrometry with guest editors Uwe Karst and Martin Vogel.

M. Vogel • U. Karst $(\bowtie)$

Institut für Anorganische und Analytische Chemie, Westfälische

Wilhelms-Universität Münster,

Wilhelm-Klemm-Str. 8,

48149 Münster, Germany

e-mail: uk@uni-muenster.de 


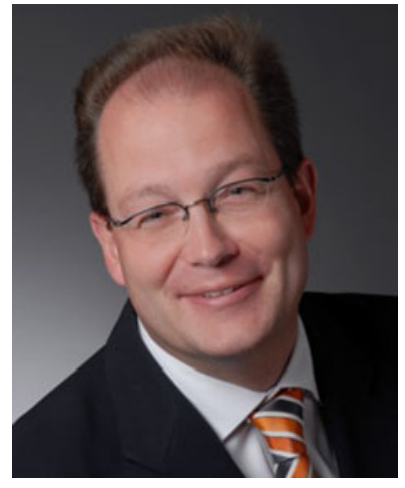

Uwe Karst

received his Ph.D. at the University of Münster, Germany, working in Karl Cammann's group, and moved to the University of Colorado in Boulder for a postdoctoral fellowship with Robert E. Sievers. After finishing his habilitation in Münster, he was appointed Full Professor of Chemical Analysis at the University of Twente (the Netherlands). In 2005, he took over his current position as Chair of Analytical Chemistry in Münster. His main research interests include hyphenated techniques, focussing in particular on pharmaceutical analysis, elemental speciation and metallomics.

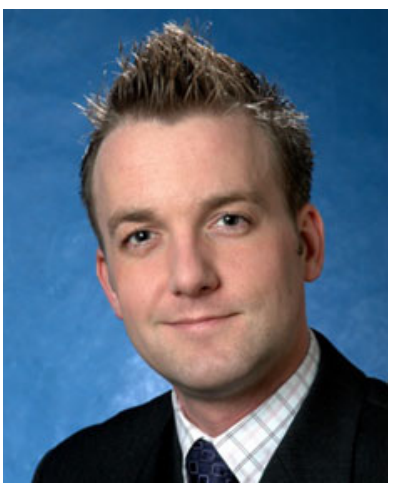

Martin Vogel

studied chemistry at the University of Münster from 1994 to 1998 and finished with a "Dipl.-Chem.". In 2001, he received a Ph.D. in analytical chemistry. His thesis work, performed under the direction of Professor Karst, was directed towards the development of a derivatisation method for the determination of airborne isocyanates utilising liquid chromatography with spectroscopic and mass spectrometric detec-

tion. Following his doctoral studies, he joined the faculty of Chemical Technology at the University of Twente (the Netherlands) as an assistant professor of analytical chemistry in 2001. Since May 2006, Martin Vogel has been a member of the Department of Analytical Chemistry at the University of Münster 